ARTÍCULO ORIGINAL

\title{
OSTEOGÉNESIS IMPERFECTA: ESTUDIO AUDITIVO Y GENÉTICO DE UNA FAMILIA CON UNA MUTACIÓN EN EL GEN COL1A1
}

\section{Osteogenesis imperfecta: Hearing and genetic study of a family with a mutation in the COL1A1 gene}

\author{
Leticia ACLE-CERVERA ${ }^{1}$; Patricia CORRIOLS-NOVAL ${ }^{1}$; María Teresa GIL-AGUILAR ${ }^{1}$; \\ Ana FONTALVA-ROMERO²; Carmelo MORALES-ANGULO ${ }^{1}$ \\ Hospital Universitario Marqués de Valdecilla \\ ${ }^{1}$ Servicio de Otorrinolaringología. ${ }^{2}$ Servicio de Genética Molecular. Santander. España \\ Correspondencia:leticia_acle@hotmail.com
}

Fecha de recepción: 7 de octubre de 2018

Fecha de aceptación: 23 de octubre de 2018

Fecha de publicación: 25 de octubre de 2018

Fecha de publicación del fascículo: 1 de junio de 2019

Conflicto de intereses: Los autores declaran no tener conflictos de intereses

Imágenes: Los autores declaran haber obtenido las imágenes con el permiso de los pacientes

Política de derechos y autoarchivo: se permite el autoarchivo de la versión post-print (SHERPA/RoMEO)

Licencia CC BY-NC-ND. Licencia Creative Commons Atribución-NoComercial-SinDerivar 4.0 Internacional

Universidad de Salamanca. Su comercialización está sujeta al permiso del editor

RESUMEN: Introducción: La osteogénesis imperfecta (OI) es una enfermedad hereditaria relacionada con la formación de tejido conectivo que se caracteriza, entre otros, por la aparición de fracturas recurrentes, escleras azules e hipoacusia. El objetivo de nuestro estudio fue demostrar la heterogeneidad clínica-auditiva en la osteogénesis imperfecta. Método: Se realizó un estudio clínico-genético de una familia de 4 miembros en la que tres de ellos padecían hipoacusia. Resultados: El estudio genético realizado a los cuatro pacientes demostró la mutación c.804+1G>A en heterocigosis en el intrón 11 del gen COL1A en los tres miembros de la familia que presentaban hipoacusia. Los tipos y severidad de la hipoacusia encontrados fueron diferentes en cada individuo: una hipoacusia neurosensorial leve bilateral, con predominio para altas frecuencias; una hipoacusia mixta bilateral moderada y la tercera un hipoacusia de transmisión unilateral leve. Conclusiones: La pérdida auditiva asociada a la mutación c.804+1G>A en el intrón 11 del gen COL1A1, presenta una gran variabilidad en cuanto al grado de afectación y severidad y también en relación al tipo de hipoacusia 


\section{OSTEOGÉNESIS IMPERFECTA: ESTUDIO AUDITIVO Y GENÉTICO DE UNA FAMILIA CON UNA MUTACIÓN EN EL GEN COL1A1 ACLE-CERVERA L ET AL}

desarrollada, que puede ser tanto neurosensorial como trasmisiva, incluso en individuos pertenecientes a la misma familia.

PALABRAS CLAVE: osteogénesis imperfecta; hipoacusia; gen COL1A1; heterogenicidad.

SUMMARY: Introduction: Osteogenesis imperfecta (OI) is a rare hereditary connective tissue disease that results in a bone fragility, blue sclerae and hearing loss. The objective of our study was to determine the heterogeneity and variety of the clinical- hearing spectrum of the OI. Methods: Genetic study was performed in a four-member family, where three of them presented hearing impairment. Results: Genetic study performed to the four patients determine c.804+1G>A heterozygosis mutation in the 11 intron of COL1A gene, in those three patients with hearing loss. Severity and type of hearing loss found were different in each patient: in one case a mild bilateral neurosensorial hearing loss, with high frequencies affected; another one bilateral moderate mixed hearing loss, and the third one was a unilateral mild conductive hearing loss. Conclusions: Hearing loss associated with c.804+1G $>$ A mutation in the 11 intron of del COL1A1 gen, is characterized by a high variability related to the severity and kind of hearing loss developed, that it could be even neurosensorial or conductive type, even in members of the same family.

KEYWORDS: osteogenesis imperfect; hearing loss; COL1A1 gene; heterogeneity.

\section{INTRODUCCIÓN}

La osteogénesis imperfecta (OI) es una enfermedad hereditaria relacionada con la formación de tejido conectivo que se caracteriza por osteopenia, fracturas recurrentes, deformidad ósea y baja estatura así como escleras azules, hiperlaxitud articular y dentinogénesis imperfecta [1]. Su prevalencia oscila entre 6-7/100.000 individuos [2]. La clasificación propuesta por Sillence distingue 4 subtipos en función del grado de afectación sistémica: I (leve), II (letal), III (severo) y IV (moderado). El grado IV es el más prevalente, representando el $85 \%$ de los casos de OI, y cuyo genotipo está asociado a una mutación que se presenta en heterocigosis en el gen COL1A1 o COL1A2, y que se transmite con una herencia autonómica dominante.

Se han descrito otras formas de OI autosómicas recesivas (tipos V-XII) que representan el $15 \%$ restante de los casos, y que se caracterizan por su mayor severidad e implicación de genes diferentes [3].

En el contexto de la OI, la hipoacusia está presente en el $37 \%$ al $64 \%$ de los pacientes y su presentación y desarrollo tiene variabilidad interfamiliar e intrafamiliar para una misma mutación [4].

El objetivo de nuestro estudio fue determinar los hallazgos clínicos que presenta una familia afecta de OI, en la que se demostró una mutación genética no descrita previamente hasta la fecha, así como demostrar la heterogeneidad clínico-auditiva relativa al genotipo incluso dentro de una misma familia con idéntica mutación.

\section{MATERIAL Y MÉTODO}

Se estudió una familia de 4 miembros, en la que la madre (I:1) y un hijo (II:2, Figura 1) presentaban diagnóstico clínico previo de OI. Tras obtener un consentimiento informado de cada paciente, se procedió a realizar una audiometría tonal (Interacoustics AD629B) doble ciego y posteriormente un estudio genético de secuenciación masiva a partir de muestras de sangre extraídas en todos ellos.

\section{RESULTADOS}

El patrón de herencia de OI en la familia estudiada resultó ser autosómico dominante (Figura 


\section{OSTEOGÉNESIS IMPERFECTA: ESTUDIO AUDITIVO Y GENÉTICO DE UNA FAMILIA CON UNA MUTACIÓN EN EL GEN COL1A1}

ACLE-CERVERA L ET AL

1). La mujer de 56 años (1:1, Figura 2) presentaba en el momento del estudio una hipoacusia mixta moderada derecha y una neurosensorial leve izquierda de predominio para altas frecuencias, sin otra sintomatología acompañante. Había sido intervenida de otosclerosis de oído izquiero 16 años antes con clara mejoría auditiva. En la tomografía computarizada (TC) de oídos se objetivaron signos incipientes de osteoporosis en el esqueleto de la base de cráneo y peñascos.

Un descendiente de 32 años fue diagnosticado de OI desde el nacimiento por anomalías congénitas del esqueleto, con escleras azules, y fracturas atípicas (de antebrazo con 7 años y de olécranon con 14 años). No presentaba hipoacusia objetiva. En la audiometría se objetivó hipoacusia neurosensorial leve bilateral en de predominio en frecuencias agudas (Figura 3 ).

Una descendiente de 29 años que presentaba escleras azules (figura 4) y había tenido unas fracturas de falanges de los pies dos años antes, refería hipoacusia derecha de larga de evolución. En la audiometría se objetivaba una hipoacusia de transmisión derecha leve con reflejo estapedial negativo, que podría ser compatible con un cuadro de otosclerosis (Figura 5). En el TAC de oídos se objetivaron ambos huesos temporales muy neumatizados asociado a desmineralización (Figura 6).

Se realizó un estudio genético de secuenciación masiva mediante next generation sequencing (NGS) en los 4 miembros de la familia. Se demostró que estos tres pacientes presentaban la mutación c.804+1G>A en heterocigosis en el intrón 11 del gen COL1A1. Esta mutación produce una ostegénesis imperfecta de fenotipo leve (tipo I en la clasificación de Sillence). El padre de la familia resultó negativo para la mutación y no presentaba niguna sintomatolgía relevante en el contexto de la OI.

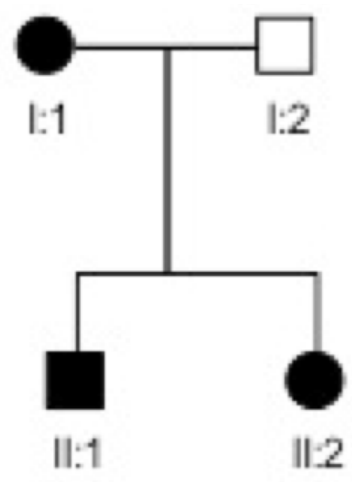

Figura 1. Árbol genealógico de la familia afectada.
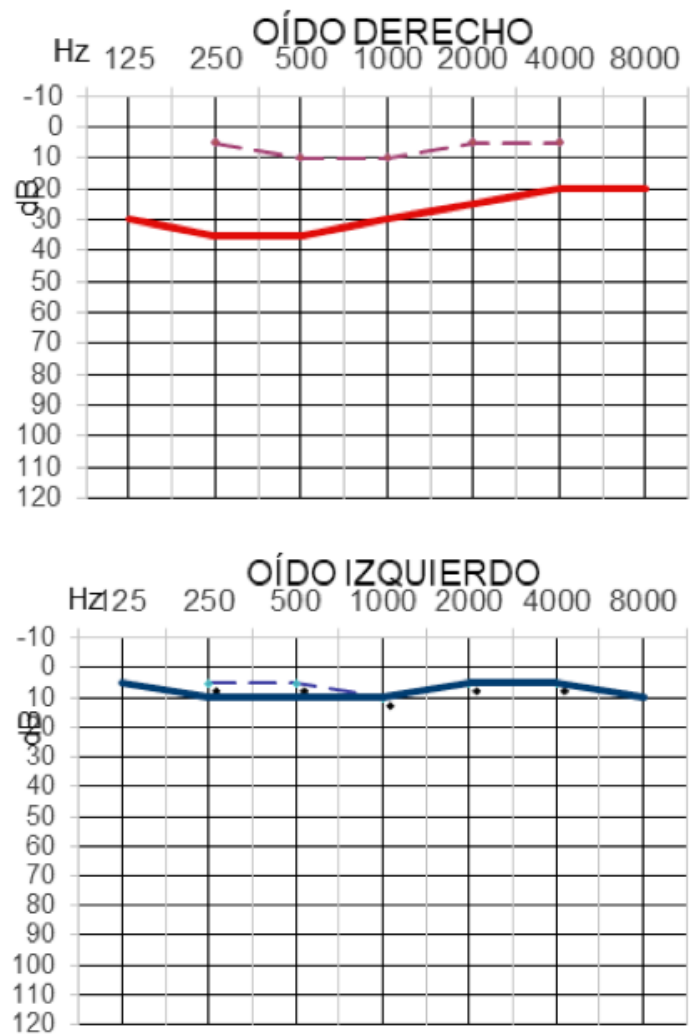

Figura 2. Hipoacusia mixta derecha moderada grado 1 con GAP medio de $20 \mathrm{~dB}$ e hipoacusia neurosensorial izquierda tras la mejoría posterior a la cirugía (I:1) 

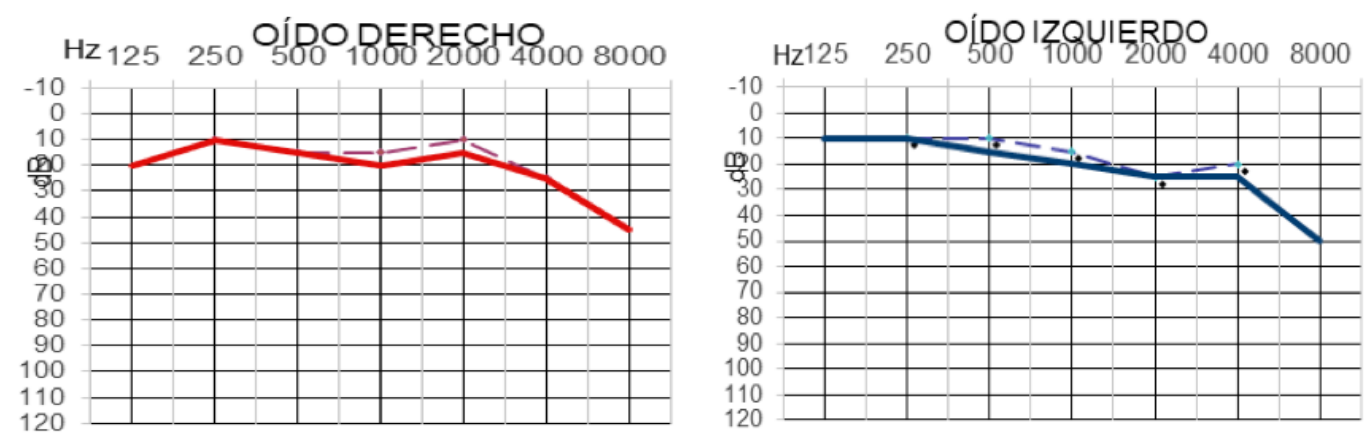

Figura 3. Hipoacusia neurosensorial bilateral leve con descenso leve en frecuencias agudas (II:1)

\section{DISCUSIÓN}

La mutación c.804+1G>A en heterocigosis en el intrón 11 del gen COL1A1 es una mutación que se trasmite con un carácter autonómico dominante y que no ha sido descrita previamente en el desarrollo de la OI, no constando su registro en las bases de datos ni en la bibliografía consultada de esta enfermedad. Se ha descrito una mutación similar c.804+1G >C en COL1A1 sin descripción del fenotipo asociado. En la mutación detectada en nuestra familia (c.804+1G>A) se produce por una sustitución de la base nitrogenada de la guanina por una adenina en el primer nucleótido del intrón número 11. El intrón es un material genético que no se trascribe para la formación proteica, si bien una mutación en los primeros nucleótidos de un intrón puede modificar el posterior ensamblaje de los exones.

En cuanto a la hipoacusia asociada a la osteogénesis imperfecta, su inicio suele darse entre la segunda y cuarta década de la vida [4]. Al inicio, se desarrolla una hipoacusia de transmisión leve que normalmente evoluciona a una hipoacusia mixta de intensidad de leve a profunda debido a una atrofia progresiva en las células ciliadas del órgano de Corti o una degeneración precoz de la estría vascular, debido a la formación de hueso anómalo dentro y alrededor de la cóclea, dónde se encuentran estas estructuras [4].

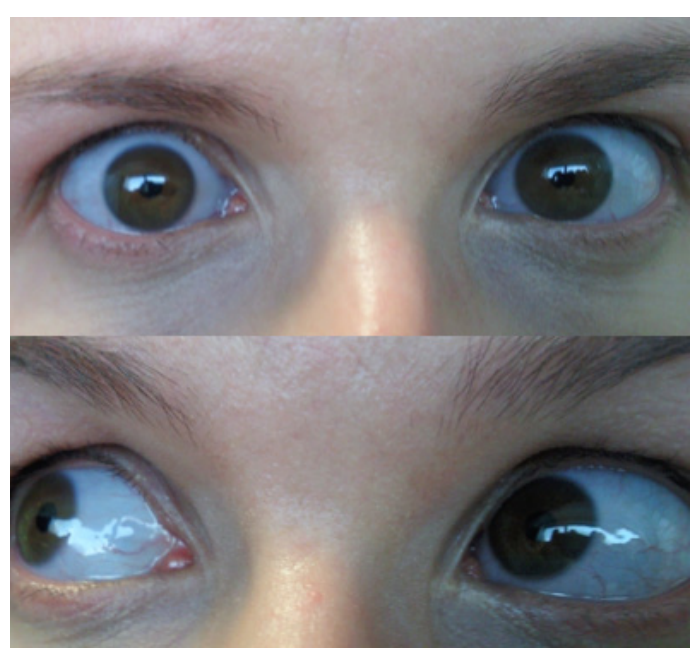

Figura 4. Escleras azules en la paciente II-2

Sin embargo, como se demuestra en nuestro estudio la hipoacusia asociada a la OI es heterogénea en el tipo, aparición y severidad en cada paciente, presentando una gran variabilidad entre familias afectadas con la misma mutación e incluso entre los pacientes de una misma familia [5].

Los hallazgos evidenciados en la TC, demuestran focos hipodensos en la fissula antefenestram (lo más frecuente), ventana oval y ventana redonda [6], así como hipodensidades retrofenestrales que afectan a las espiras cocleares, canal del nervio facial, o canales semicirculares, o incluso simular 

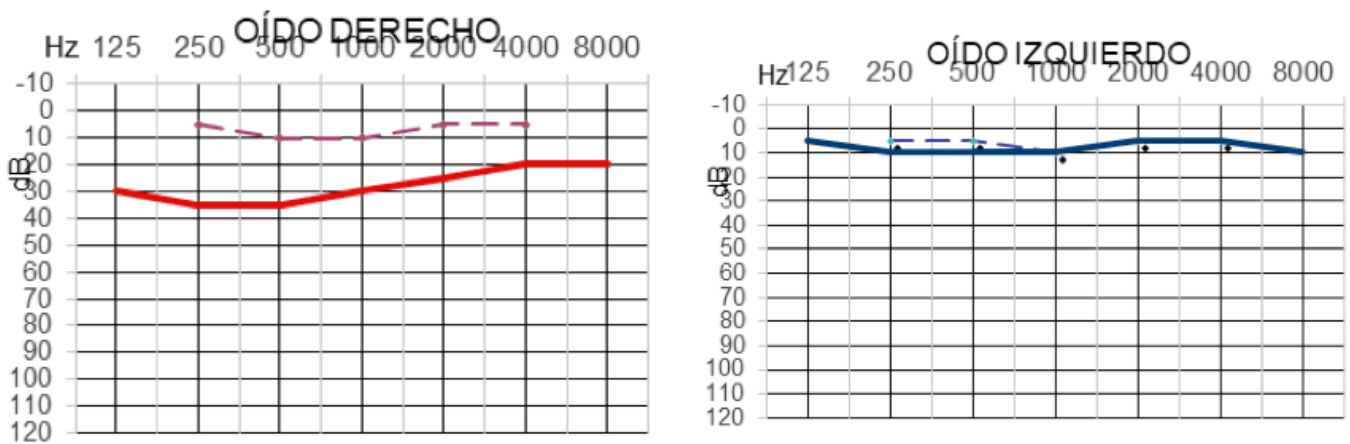

Figura 5. Hipoacusia de trasmisión derecha leve con GAP medio de 15dB. Normoacusia izquierda (II:2)

una cuarta espira coclear en un tercio de los casos [6]. En un $72 \%$ de los pacientes la localización de la hipodensidad se correlaciona con el tipo de sordera [6].

Por otra parte, aunque en los pacientes de nuestro estudio no se llevó a cabo dicha técnica diagnóstica, el estudio mediante resonancia magnética con gadolinio, puede mostrar lesiones activas en pacientes con hipoacusia mixta [6].

Los pacientes que presentan una hipoacusia de transmisión con un gap significativo entre la vía ósea y aérea, son susceptibles de ser intervenidos quirúrgicamente mediante la realización de una estapedectomía o estapedotomía, consiguiendo unos umbrales auditivos aceptables [7], como ocurrió en la progenitora de la familia estudiada. En casos de hipoacusias mixtas, la combinación de implantes auditivos como el Vibrant Sound bridge junto con la realización de la estapedotomía ha demostrado óptimos resultados en un grupo reducido de pacientes con OI [8].

En pacientes con OI e hipoacusia profunda con un insuficiente rendimiento audioprotésico, el implante coclear puede estar indicado. Han sido descritos casos de estimulación del nervio facial a través del implante coclear que han requerido la inutilización de algunos de sus electrodos[9]. A pesar de algunas complicaciones, el implante coclear aporta un valioso beneficio en pacientes con osteogénesis imperfecta que sufren hipoacusia severa profunda $[10,11]$.

Ediciones Universidad de Salamanca / @®@

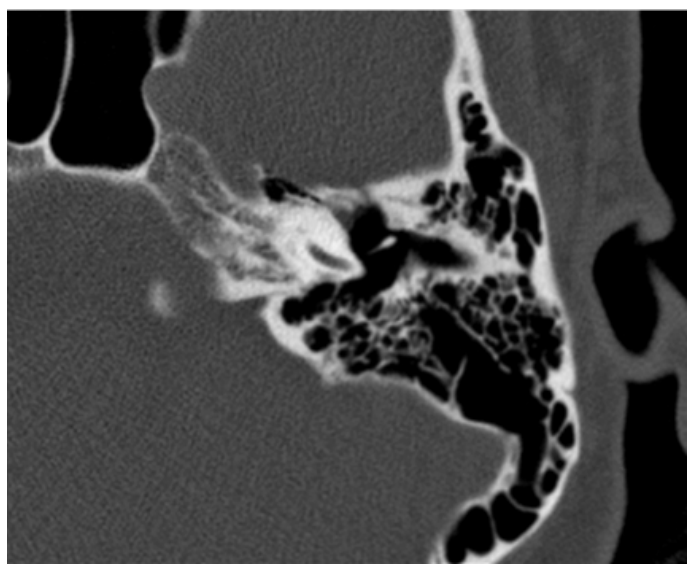

Figura 6. Corte axial de TAC oído izdo. Desmineralización del peñasco

Por otra parte, estudios recientes sugieren que el tratamiento con bifosfonatos puede mejorar la historia natural de la enfermedad, si se realiza tempranamente en niños con osteogénesis imperfecta [12]. El tratamiento oral con alendronato ha demostrado un incremento en la densidad mineral ósea (DMO) sin producir mejoría a nivel auditivo en pacientes adultos con osteogénesis imperfecta [13]. La terapia con hormona del crecimiento humano como complemento de la terapia con bifosfonatos demostró un aumento de la DMO y un crecimiento lineal mejorado [14]. 


\section{OSTEOGÉNESIS IMPERFECTA: ESTUDIO AUDITIVO Y GENÉTICO DE UNA FAMILIA CON UNA MUTACIÓN EN EL GEN COL1A1 ACLE-CERVERA L ET AL}

\section{CONCLUSIONES}

La pérdida auditiva que presentan las familias con OI y la mutación c.804+1G>A en el intrón 11 del gen COL1A1, se caracteriza por una gran variabilidad en cuanto al grado de severidad y también en relación al tipo de hipoacusia desarrollada, que puede ser tanto neurosensorial como transmisiva o mixta.

\section{BIBLIOGRAFÍA}

1. Baujat G, Lebre AS, Cormier-Daire V, Le Mener M. Osteogenesis imperfecta; diagnosis information (clinical and genetic classification). Arch Pediatr. 2008;15:789-91.

2. Amor MB, Glorieux FH., Rauch F. Osteogenesis imperfecta. Best Pract Res Clin Rheumatol. 2008;22:85-100.

3. Marini J, Smith SM . Osteogenesis imperfecta. MDText.com, Inc. 2000.

4. Swinnen F., Coucke PJ. De Paepe AM, Symoens $\mathrm{S}$, Malfait F, Gentile FV, et al. Osteogenesis imperfecta: the audiological phenotype lacks correlation with the genotype. Orphanet Journal of Rare Diseases. 2011;6:88-

5. Pereira da Silva A, Feliciano T, Figueiringhas R, Almeida E, Sousa C. Osteogenesis imperfecta and hearing loss, description of three case reports. Acta Otorrinolaringol Esp. 2013;64:413-7.

6. Swinnen FK, Casselman JW, De Leenheer EM, Cremers CW, Dhooge IJ. Temporal bone imaging in osteogenesis imperfecta patients with hearing loss. Laryngoscope. 2013;123:1988-95.
7. Vincent R, Wegner I, Stegenon I, Grolman W. Stapedotomy in osteogenesis imperfecta: a prospective study of 32 consecutive cases. Otol Neurotol. 2014;35;1785-9.

8. G. Kontorinis, T. Lenarz, H. Mojallal, A. L. Hinze, and B.Schwab, "Power stapes: an alternative method for treating hearing loss in osteogenesis imperfecta?" Otology and Neurotology, vol. 32, no. 4, pp. 589-595, 2011.

9. Makizumi Y, Kashio A, Sakamoto T, Karino S, Kakigi A, Iwasaki S, et al. Cochlear implantation in a patient with osteogenesis imperfecta. Auris Nasus Larynx. 2013;40:510-513.

10. Pillion,J, Vernick, D, Shapiro J. Hearing Loss in Osteogenesis Imperfecta: Characteristics and Treatment Considerations. Genetics Research International. Volume 2011, Article 983942.

11. Migirov L, Henkin Y, Hildesheimer M, Kronenberg J. Cochlear implantation in a child with osteogenesis imperfecta. International Journal of Pediatric Otorhinolaryngology. 2003;67:677-80.

12. Ting $\mathrm{TH}$, Zacharing MR. Hearing in bisphosphanate-treated children with osteogenesis imperfecta. Our experience in thirty six young patients. Clinc Otolaryngol. 2012;37:229-33.

13. Chevrel G, Schott AM, Fontanges E, Charrin JE, Lina-Granade G,. Effects of oral alendronate on BMD in adult patients with osteogenesis imperfecta: a 3-year randomized placebo-controlled trial. J Bone Miner Res. 2006;21:300-6

14. Steiner,R, Adsit,J and Basel, D. COL1A1/2-Related Osteogenesis Imperfecta. In: Adam MP, Ardinger $\mathrm{HH}$, Pagon RA, Wallace SE, Bean LJH, Stephens $\mathrm{K}$, Amemiya A, editors. GeneReviews ${ }^{\circ}$. Seattle (WA): University of Washington, Seattle; 19932018. 